13

\title{
Об эффекте Фарадея в магнитных коллоидных наносистемах
}

\author{
(С Ю.И. Диканский, С.Д. Туркин
}

Северо-Кавказский федеральный университет,

Институт математики и естественных наук,

355029 Ставрополь, Россия

e-mail: Dikansky@mail.ru

(Поступило в Редакцию 1 марта 2017 г. В окончательной редакции 3 мая 2017 г.)

\begin{abstract}
Проведено экспериментальное исследование распространения плоско поляризованной электромагнитной волны СВЧ-диапазона в магнитных коллоидах с магнетитовыми частицами и магниточувствительных эмульсиях, синтезированных на их основе. Установлено, что воздействие магнитного поля, направленного вдоль распространения волны, на такие среды приводит к повороту плоскости поляризации волны (эффекту Фарадея), при этом проявление этого эффекта начинается только при превышении некоторого критического значения объемной концентрации магнетита. Обнаружены особенности этого эффекта в структурированных магнитных коллоидах и магнитных эмульсиях, связанные с изменениями их структуры при воздействии магнитного поля.
\end{abstract}

DOI: $10.21883 / J T F .2017 .12 .45217 .2222$

\section{Введение}

Эффект Фарадея, обнаруженный в ферритах в СВЧ диапазоне нашел широкое применение радиолокационной технике и радиорелейной передаче информации [1]. Оказалось, что этот эффект может проявляться не только в твердых, но в жидких коллоидах ферритов - так называемых магнитных жидкостях [2]. Можно предположить, что его проявление в жидких средах может найти новые возможности для развития технических средств диапазона СВЧ. Магнитные жидкости - коллоидные растворы ферро- и ферримагнетиков в немагнитных жидких средах - сочетают уникальные свойства текучести со способностью намагничиваться. В настоящее время основные физические свойства магнитных жидкостей (магнитные, электрофизические, магнитооптические) считаются достаточно хорошо изученными, подробное описание результатов исследования которых можно найти в монографиях [3-5]. В ряде работ (например, [6-9]) исследовалось взаимодействие таких систем с электромагнитным СВЧ излучением, однако его особенности остаются к настоящему времени недостаточно полно изученными. В связи с этим в настоящей работе были предприняты исследования особенностей распространения электромагнитных волн диапазона СВЧ в высокодисперсных коллоидах магнетита при воздействии постоянного магнитного поля.

\section{Методика измерений и образцы для исследований}

Исследования проводились на частоте электромагнитных волн $11 \mathrm{GHz}$, генерируемых диодом Ганна. На рис. 1 представлена схема используемой экспериментальной установки. Плоско-поляризованное излучение направлялось в круглый волновод длина длиной $20 \mathrm{~cm}$, в кото- ром располагалась кювета с образцом эллипсоидальной формы (длина кюветы составляла $6 \mathrm{~cm}$, диаметр ее цилиндрической части $-2.7 \mathrm{~cm})$. Интенсивность этого излучения регистрировалась детектором, представлявшим собой также диод Ганна, встроенный в короткий прямоугольный волновод. Приемный диод располагался на платформе, благодаря возможности вращения которой,

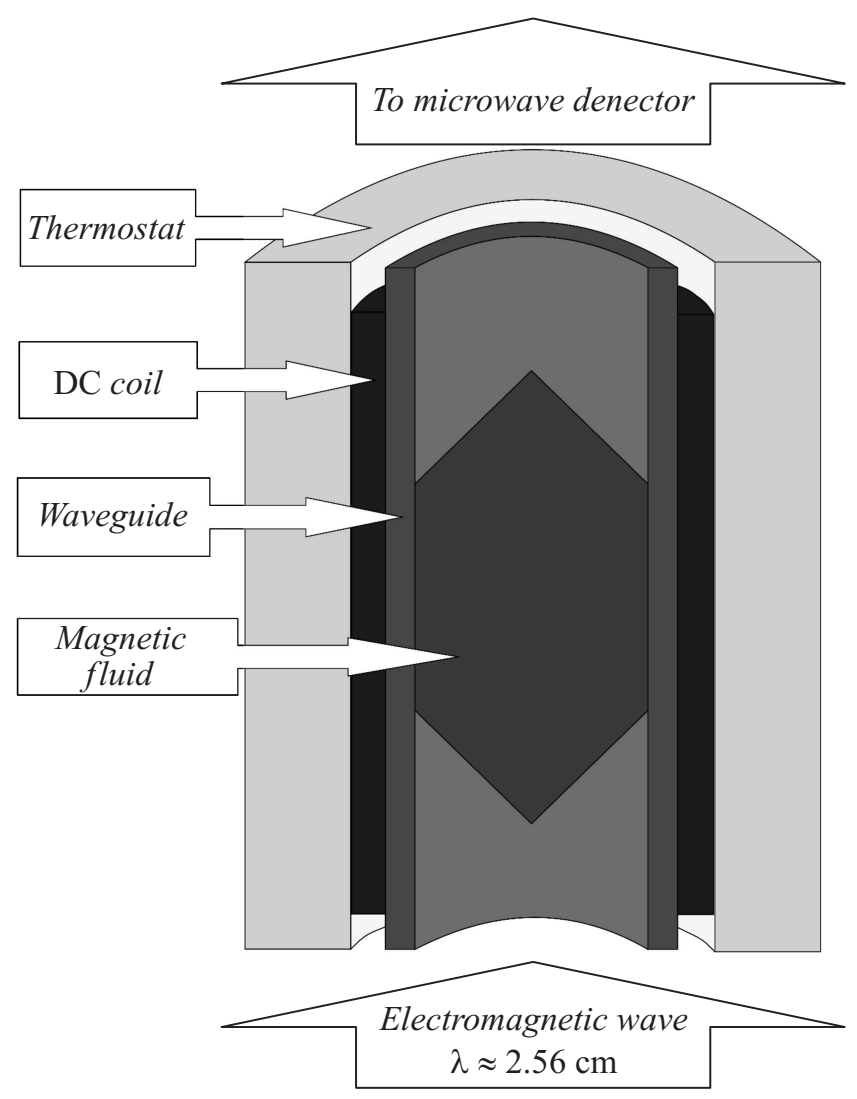

Рис. 1. Схема экспериментальной установки. 


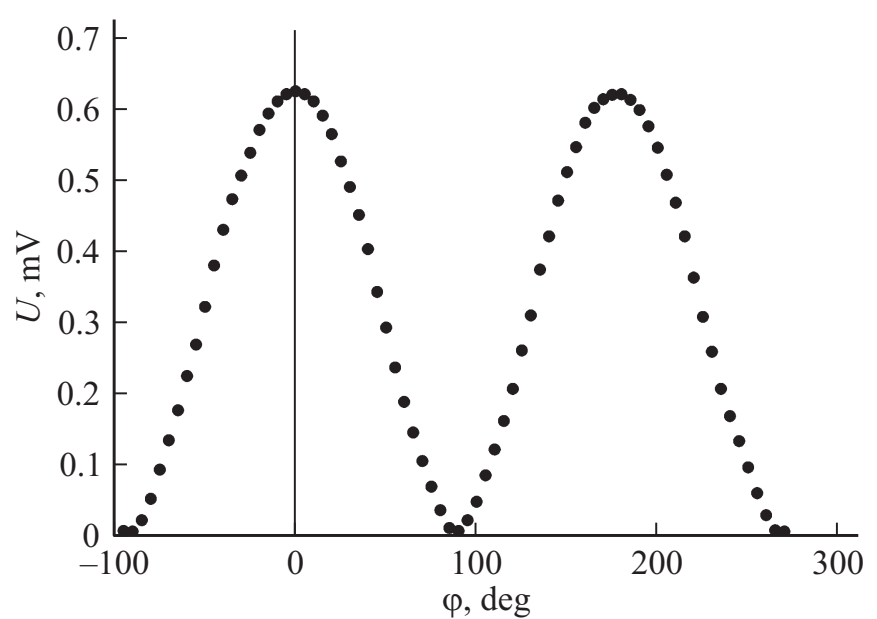

Pис. 2. Изменение сигнала с датчика при его повороте на один оборот вокруг оси (при отсутствии образца).

он вместе с волноводом мог поворачиваться вокруг оси, совпадающей с направлением распространения излучения. Отчет угла поворота осуществлялся с помощью лимба с ценой деления 0.01 градуса. Распределение напряжения принимаемой ЭМ волны (при отсутствии в волноводе кюветы с образцом) при повороте датчика на 1 оборот вокруг оси представлено на рис. 2. Внешнее постоянное магнитное поле, направленное вдоль волновода, с максимальной напряженностью $38 \mathrm{kA} / \mathrm{m}$ создавалось с помощью соленоида. Для предотвращения температурных флуктуаций система термостатировалась.

Для проведения исследований были использованы 4 образца высокодисперсного магнетита. Образец № 1 представлял собой магнитный коллоид на основе керосина с магнетитовыми однодоменными частицами, средний диметр которых составлял $9 \mathrm{~nm}$. В качестве стабилизатора коллоидной системы использовалась олеиновая кислота, исходная объемная концентрация дисперсной фазы составляла 18\%. Образец, как можно судить по наблюдениям в оптический микроскоп, представлял собой однородную среду без видимых включений и агрегатов из коллоидных частиц.

Кроме однородного (неагрегированного) магнитного коллоида для исследований был использован магнитный коллоид, содержащий хорошо развитую систему микрокапельных агрегатов (образец № 2). Он был получен из высококонцентрированного образца магнитной жидкости на основе керосина с достаточно крупными частицам (средним диаметром $13 \mathrm{~nm}$ ), путем разбавления его керосином до концентрации дисперсной фазы $6.5 \%$. Кроме того, были проведены исследования полученного таким же образом образца с концентрацией дисперсной фазы 2.5\%. Воздействие магнитного поля на эти образцы приводит к деформации (вытягиванию) микрокапельных агрегатов с дальнейшим объединением их в вытянутые структуры и созданию упорядоченной структурной решетки (рис. 3,a), которая разрушается при выключении
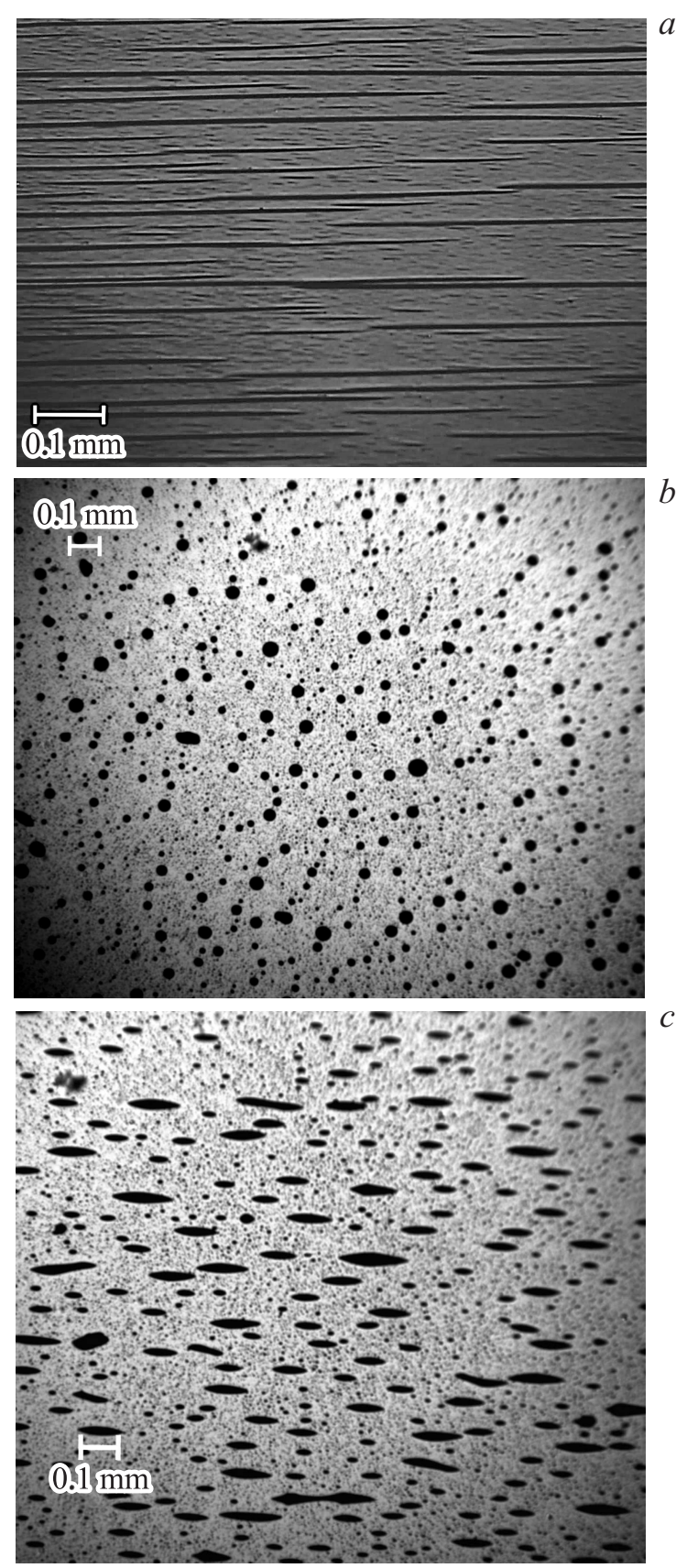

Рис. 3. Структура образцов № 2 и № 3 ( $a$ - структура образца № 2 при воздействии на него внешнего магнитного поля напряженностью $H_{0}=32 \mathrm{kA} / \mathrm{m}, b$ и $c-$ структура образца № 3 без и при воздействии внешнего магнитного поля напряженностью $H_{0}=32 \mathrm{kA} / \mathrm{m}$ соответственно).

поля. Образец № 3 был получен диспергированием образца № 1 в несмешивающемся с ним масле АМГ-10 и представлял собой эмульсию капель магнитой жидкости в немагнитной среде с концентрацией магнитной фазы $6.7 \%$.

Образец № 4 также представлял собой магнитную эмульсию, которая в отличие от образца № 3 была получена эмульгированием магнитной жидкости на по- 
лиэтилсилаксановой основе (с объемной концентрацией дисперсной фазы 6\%) в масле АМГ-10. Объемное содержание магнетита в последней эмульсии составляло $2.3 \%$. Воздействие магнитного поля на образцы № 3 и № 4 приводит к деформации первоначально сферических микрокапель вдоль направления поля, которые приобретают первоначальную сферическую форму при его выключении (рис. $3, b, c)$.

\section{Экспериментальные результаты и их обсуждение}

Было обнаружено, что воздействие постоянного магнитного поля, направленного вдоль направления распространения волны в магнитных коллоидах приводит к повороту плоскости ее поляризации, угол которого зависит от напряженности поля. В качестве иллюстрации этого на рис. 4 представлены зависимости сигнала приемного детектора от угла его поворота вокруг оси волновода с образцом № 1 при отсутствии магнитного поля (кривая 1 ) и в магнитном поле напряженностью $32 \mathrm{kA} / \mathrm{m}$ (кривая 2), а на рис. 5 - зависимость угла поворота плоскости поляризации волны, прошедшей через этот образец, от напряженности магнитного поля. Как видно из графика, последняя зависимость близка к линейной в области малых значений напряжености поля. Отметим, что такой же характер имеет и аналогичная зависимость для сухого порошка магнетита, полученного выпариванием дисперсионной среды из магнитного коллоида на основе керосина.

Как известно [10], в присутствии постоянного магнитного поля вектор намагниченности прецессирует вокруг вектора магнитного поля с частотой $\omega_{0}$, что описывается

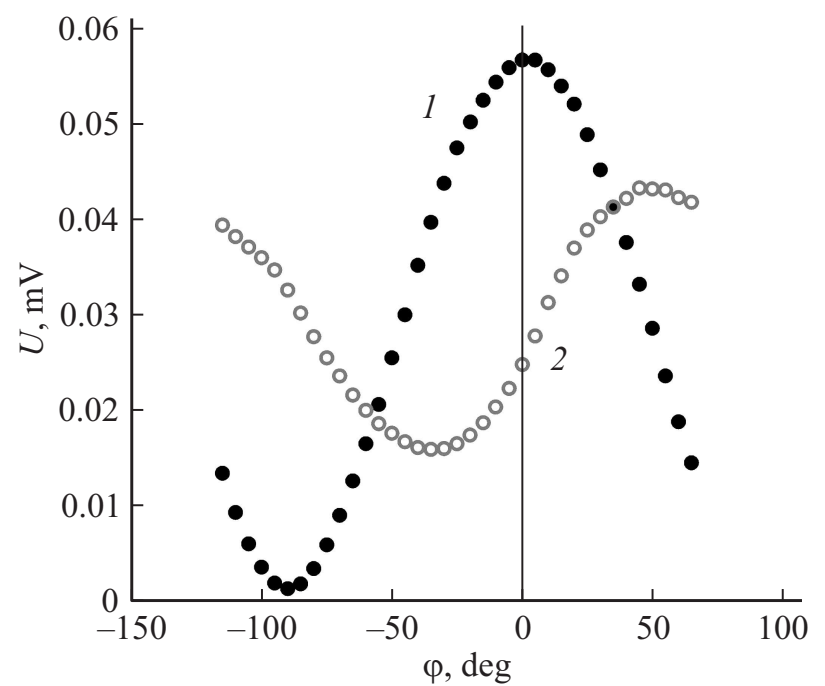

Pис. 4. Зависимости напряжения на датчике от угла поворота последнего при исследовании образца № 1 с концентрацией магнетика $18 \%$. Кривая 1 - при отсутствии магнитного поля, кривая 2 - в магнитном поле напряженностью $32 \mathrm{kA} / \mathrm{m}$.

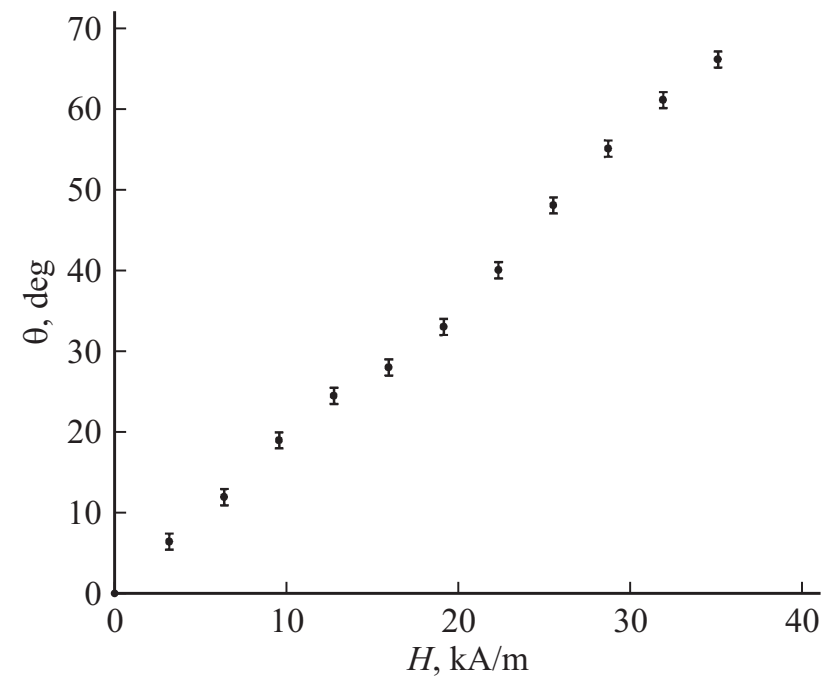

Рис. 5. Зависимость угла поворота плоскости поляризации проходящей волны через образец № 1 от напряженности внешнего постоянного магнитного поля.

следующим уравнением:

$$
\frac{d \mathbf{M}}{d t}=\mu_{0} \gamma(\mathbf{H} \times \mathbf{M})-\frac{\mu_{0} \gamma}{|\mathbf{M}|} \alpha(\mathbf{M} \times(\mathbf{H} \times \mathbf{M})),
$$

где $\mathbf{M}$ - результирующее магнитное поле, образованное из продольного постоянного внешнего поля $H_{0}$ и переменного поля электромагнитной волны, М результирующая намагниченность, $\gamma-$ гиромагнитное отношение, $\alpha-$ феноменологическая константа, описывающая затухание.

Согласно теории распространения электромагнитных волн СВЧ в ферритах [11], для исследования распространения линейно поляризованной волны ее следует разложить на две с левой и правой круговой поляризацией с различными постоянными распространения. Причем для правого вращения из решения уравнения (1), учитывая $\omega_{0}<\omega$ и $\omega_{M}<\omega$ (область малых значений напряжености поля), следует, что магнитная проницаемость равна

$$
\sqrt{\mu_{+}} \approx \sqrt{\mu_{0}}\left(1-\frac{\omega_{M}}{2 \omega}\right),
$$

а для левого вращения:

$$
\sqrt{\mu_{-}} \approx \sqrt{\mu_{0}}\left(1+\frac{\omega_{M}}{2 \omega}\right)
$$

где $\omega-$ частота электромагнитной волны, $\omega_{M}=\mu_{0} \gamma M_{0}$ и $\omega_{0}=\mu_{0} \gamma H_{0}$.

В связи с этим поворот плоскости поляризации происходит на угол

$$
\theta \approx \frac{l}{2}\left(\omega \sqrt{\mu_{+} \varepsilon}-\omega \sqrt{\mu_{-} \varepsilon}\right)=\frac{l}{2} \mu_{0} \gamma H_{0} \sqrt{\mu_{0} \varepsilon},
$$

где $l$ - расстояние, пройденное волной в феррите. 


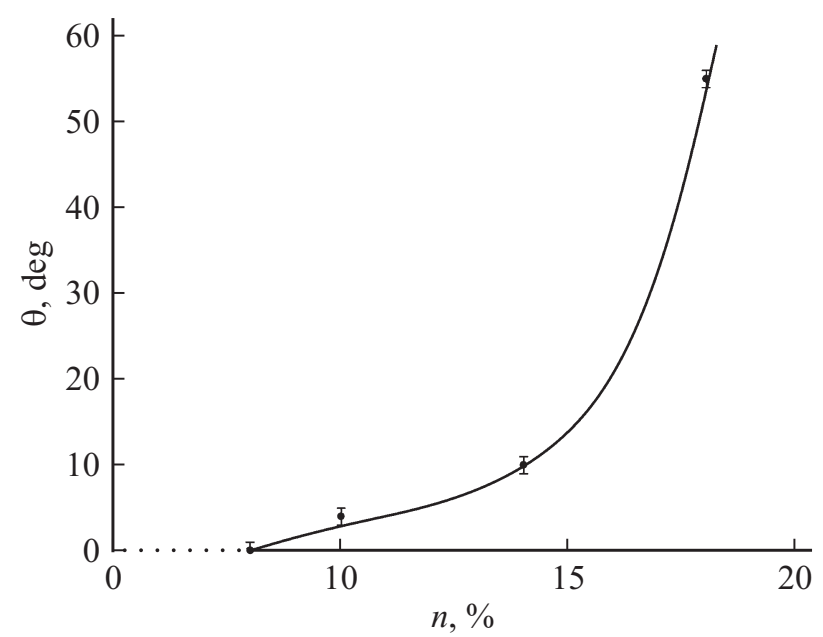

Pис. 6. Зависимость угла поворота плоскости поляризации от объемной концентрации магнетика в образце № 1 при фиксированном значении внешнего поля $H_{0}=32 \mathrm{kA} / \mathrm{m}$.

Как следует из формулы (4), угол поворота плоскости поляризации составляет с направлением магнитного поля правовинтовую систему, т.е. при изменении направления вектора $H_{0}$ на обратное плоскость поляризации будет поворачиваться в противоположную сторону на то же значение угла. Кроме того, поворот плоскости поляризации может иметь значительную величину уже при небольших постоянных полях, что согласуется с результатами эксперимента. Вместе с тем были обнаружены особенности проявления этого эффекта, характерные, по-видимому, только для дисперсных систем ферритов. Так, оказалось, что эффект Фарадея возникает только при достижении некоторого порогового значения концентрации объемной фазы. Кроме того, зависимость угла поворота плоскости поляризации при воздействии поля от объемной концентрации в интервале концентраций, превышающих пороговое значение, не является линейной (рис. 6). Можно предположить, что исчезновение эффекта при уменьшении концентрации до некоторого значения связано с локальным уменьшением количества активного вещества в образце, что вследствие малости линейного размера дисперсных частиц приводит к дифрагированию проходящей волны и прекращению ее взаимодействия с веществом. Следует также обратить внимание на общее уменьшение интенсивности сигнала при включении магнитного поля. По-видимому, это связано либо с усилением поглощения, либо с частичной деполяризацией распространяющейся в этом образце волны в случае воздействия на него магнитного поля.

Исследования, проведенные с использованием образца № 2, содержащего агрегаты, показали, что в нем также наблюдается эффект Фарадея (рис. 7), однако в этом случае интенсивность волны, прошедшей через образец, подверженный действию магнитного поля, имеет более высокое значение, чем при отсутствии последнего. Кроме того, пороговое значение объемного содержания магнетита, при котором начинает проявляться эффект, существенно ниже, чем для однородного образца. Так, в образце № 2 эффект еще заметно проявляется при объемной концентрации магнетита, равной $2.5 \%$, тогда как в образце № 1 эффект перестает регистрироваться при концентрациях магнетита, меньших 10\%. При этом зависимость угла поворота плоскости поляризации волны от напряженности поля близка к линейной только при относительно малых значениях напряженности поля (рис. 8). Очевидно, эти особенности связаны с структурным состоянием дисперсной системы и его изменением в магнитном поле.

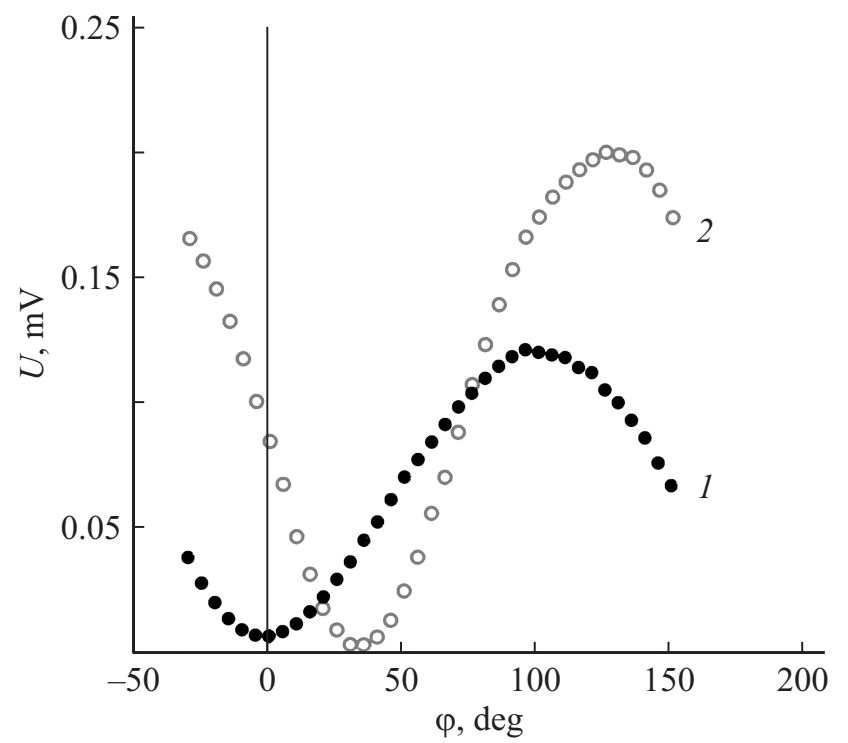

Рис. 7. Зависимости выходящего напряжения с датчика от угла поворота последнего при исследовании образца № 2. Кривая 1 - при отсутствии магнитного поля, кривая 2 в магнитном поле напряженностью $32 \mathrm{kA} / \mathrm{m}$.

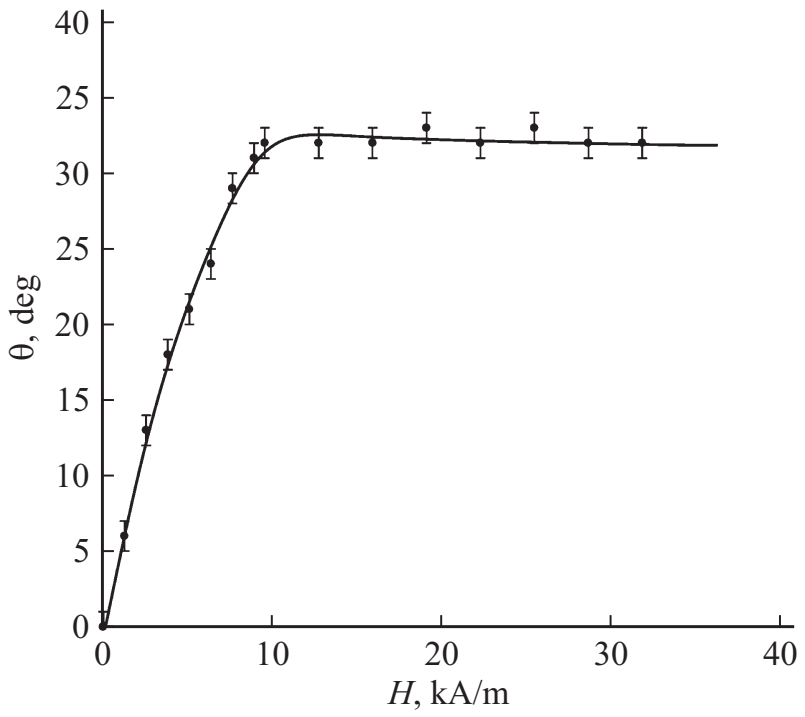

Рис. 8. Зависимость угла поворота плоскости поляризации проходящей волны через образец № 2 (объемная концентрация магнетика $6.5 \%$ ) от внешнего постоянного магнитного поля. 


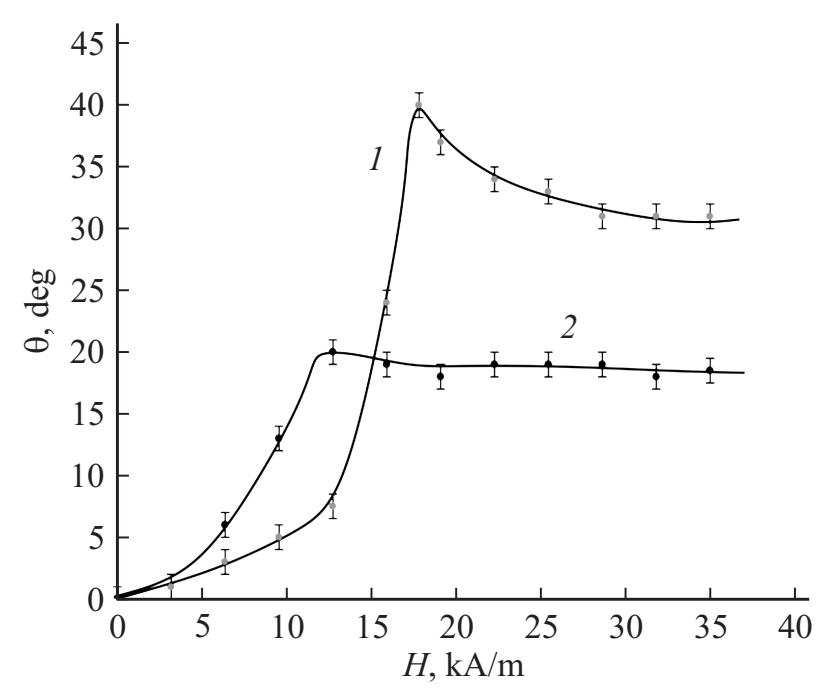

Pис. 9. Зависимости угла поворота плоскости поляризации волны, прошедшей через образцы № 3 и № 4, от напряженности внешнего постоянного магнитного поля (кривые 1 и 2 соответственно.

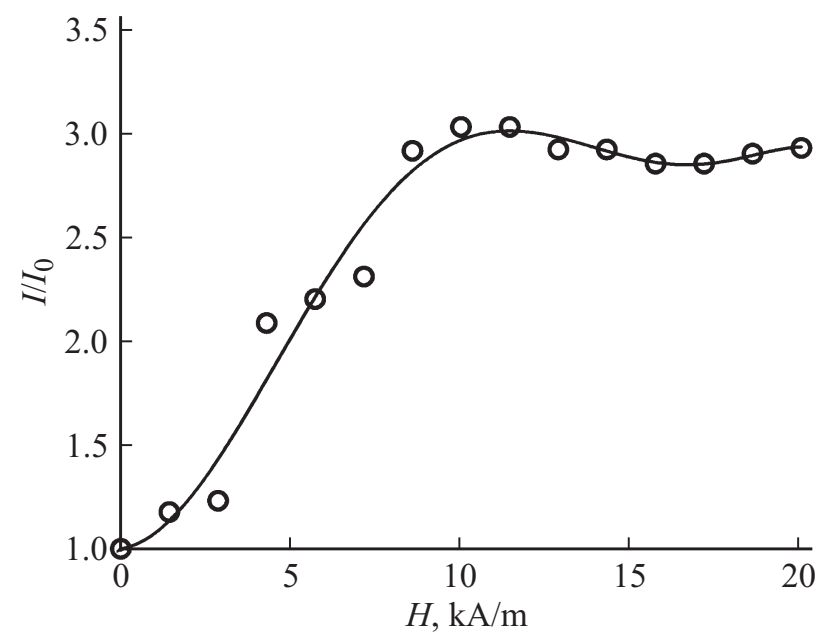

Рис. 10. Зависимость относительного изменения интенсивности анизотропного света тонким слоем образца № 4 от напряженности внешнего постоянного магнитного поля.

Увеличение сигнала на выходе детектора при воздействии на образец № 2 поля, а также более отчетливый вид экстремумов могут быть связаны с структурным изменением системы - трансформацией первоначально сферических микрокапельных агрегатов в вытянутые структуры и в последующем их некоторым пространственным упорядочением. Можно предположить, что при отсутствии внешнего поля микрокапельные агрегаты распределены по всему объему образца, при этом площадь эффективного сечения рассеяния будет пропорциональна квадрату среднего радиуса капель $r^{2}$. При включении поля происходит вытягивание капель вдоль силовых линий, что, в свою очередь, уменьшает эффек- тивную площадь сечения рассеяния, а это приводит к увеличению значения принимаемого сигнала.

С целью обоснования этого предположения были проведены исследования эффекта Фарадея в магнитных эмульсиях (образцы № 3 и № 4), в которых также наблюдаются аналогичные структурные изменения при воздействии магнитного поля.

На рис. 9 показаны зависимости угла поворота плоскости поляризации волны, прошедшей через образцы № 3 и № 4, от напряженности приложенного поля (кривые 1 и 2 соответственно). Как можно видеть из рисунка, представленные зависимости для эмульсий не являются линейными даже в области малых значений напряженности поля, кроме того, наблюдаются максимумы при достижении некоторого ее значения. Это может свидетельствовать в пользу предположения о взаимосвязи наблюдаемого эффекта с структурным состоянием системы, изменяющимся е под воздействием поля. Оценка характера структурных изменений системы при воздействии поля была проведена на основе результатов исследования анизотропного рассеяния света. На рис. 10 приведена зависимость относительной величины интенсивности рассеянного света тонким слоем образца № 4 от напряженности поля, направленного вдоль плоскости слоя (угол рассеяния составлял $\sim 1$ градус). Как можно видеть из рисунка, характер представленной зависимости качественно согласуется с зависимостью угла поворота плоскости поляризации от напряженности поля для исследованных эмульсий.

\section{Заключение}

Таким образом, проведенные исследования показали, что проявление эффекта Фарадея в коллоидных растворах ферритов имеет ряд особенностей, связанных с неоднородностью таких сред и структурными изменениями, происходящими при воздействии магнитных полей. Так, он становится заметным только при объемном содержании дисперсной фазы выше некоторого порогового значения, при этом зависимость угла поворота плоскости поляризации при воздействии поля от объемной концентрации не является линейной. В коллоидах, содержащих микрокапельные агрегаты, а также в образцах, представляющих эмульсию магнитных коллоидов в немагнитной среде, пороговое значение объемной концентрации, при которой начинает наблюдаться поворот плоскости поляризации волны под воздействием магнитного поля, имеет более низкое значение, чем в однородных (не агрегированных) коллоидах. Кроме того, характер зависимости угла поворота плоскости поляризации волны от напряженности приложенного магнитного поля в таких средах существенно отличается от аналогичной зависимости в однородных коллоидах, что связано с особенностью структурного состояния этих образцов и его изменением при воздействии магнитных полей. Очевидно, для успешного анализа обнаруженных 
особенностей требуется накопление дополнительного экспериментального и теоретического материала, что и будет являться предметом дальнейших исследований.

\section{Список литературы}

[1] Гуревич А.Г. Ферриты на сверхвысоких частотах. М.: Физматлит, 1960. $408 \mathrm{c.}$

[2] Maiorov M.M. // J. Magnetism and Magnetic Materials. 2002. P. 111-113.

[3] Розенцвейг Р. Феррогидродинамика. М.: Мир. 1989. 467 с.

[4] Фертман B.E. Магнитные жидкости. Минск: Вышэйшая школа, 1988. $184 \mathrm{c}$.

[5] Блум Э.Я., Майоров М.М., Цеберс А.О. Магнитные жидкости. Рига: Зинатне, 1986. 386 с.

[6] Усанов Д.А., Скрипаль Ал.В., Скрипаль Ан.В., Постельга А.Э. // ЖТФ. 2009. Т. 79. Вып. 9. С. 146-148.

[7] Усанов Д.А., Скрипаль Ал.В., Скрипаль Ан.В., Курганов А.В. // ЖТФ. 2001. Т. 71. Вып. 12. С. 26-29.

[8] Усанов Д.А., Постельга А.Э., Алтынбаев С.В. // ЖТФ. 2013. Т. 83. Вып. 11. С. 30-33.

[9] Усанов Д.А., Скрипаль Ал.В., Скрипаль Ан.В., Постельга А.Э., Райхер Ю.Л., Степанов В.И. // ЖТФ. 2006. Т. 76. Вып. 11. С. 126-129.

[10] Ландау Л.Д., Лифиии, Е.М. Теоретическая физика. Электродинамика сплошных сред. 2-е изд. М.: Наука. Гл. ред. Физматлит, 1982. $621 \mathrm{c.}$

[11] Карлинер М.М. Электродинамика СВЧ. Курс лекций. 2-е изд. Новосибирск, 2006. 258 с. 